Research article

\title{
Selenoprotein $\mathbf{N}$ is dynamically expressed during mouse development and detected early in muscle precursors Perrine Castets ${ }^{1,2,6}$, Svetlana Maugenre ${ }^{1,2,6}$, Corine Gartioux ${ }^{1,2,7}$, Mathieu Rederstorff 3,8 , Alain $\mathrm{Krol}^{3}$, Alain Lescure ${ }^{3}$, Shahragim Tajbakhsh ${ }^{4}$, Valérie Allamand ${ }^{1,2,7}$ and Pascale Guicheney*1,2,5,6
}

Address: ${ }^{1}$ Inserm, U582, F-75013 Paris, France, ${ }^{2}$ UPMC Univ Paris 06, UMR_S582, Institut de Myologie, IFR14, F-75013 Paris, France, ${ }^{3}$ Architecture et Réactivité de l'ARN, Université de Strasbourg, CNRS, IBMC, F-67084 Strasbourg, France, ${ }^{4}$ CNRS, URA 2578, Institut Pasteur, F75015 Paris, France, ${ }^{5}$ AP-HP, Groupe Hospitalier Pitié-Salpêtrière, Service de Biochimie Métabolique, F-75013 Paris, France, 6 Inserm, U956, UPMC Univ Paris 06, UMR_S956, F-75013 Paris, France, 7Inserm, U974, Institut de Myologie, CNRS UMR7215, UPMC Univ Paris 06, UMR_S974, IFR14, F-75013 Paris, France and ${ }^{8}$ Innsbruck Medical University, Biocenter, Section for Genomics and RNomics, A-6020 Innsbruck, Austria

Email: Perrine Castets - p.castets@institut-myologie.org; Svetlana Maugenre - svetlana.maugenre@upmc.fr;

Corine Gartioux - c.gartioux@institut-myologie.org; Mathieu Rederstorff - Mathieu.Rederstorff@i-med.ac.at; Alain Krol - a.krol@ibmc.ustrasbg.fr; Alain Lescure - a.lescure@ibmc.u-strasbg.fr; Shahragim Tajbakhsh - shaht@pasteur.fr; Valérie Allamand - v.allamand@institutmyologie.org; Pascale Guicheney* - pascale.guicheney@upmc.fr

* Corresponding author

Published: 22 August 2009

BMC Developmental Biology 2009, 9:46 doi:|0.| |86/|47|-2/3X-9-46
Received: 2 February 2009

Accepted: 22 August 2009

This article is available from: http://www.biomedcentral.com/I47I-2/3X/9/46

(C) 2009 Castets et al; licensee BioMed Central Ltd.

This is an Open Access article distributed under the terms of the Creative Commons Attribution License (http://creativecommons.org/licenses/by/2.0), which permits unrestricted use, distribution, and reproduction in any medium, provided the original work is properly cited.

\begin{abstract}
Background: In humans, mutations in the SEPNI gene, encoding selenoprotein $N(S e I N)$, are involved in early onset recessive neuromuscular disorders, referred to as SEPNI-relatedmyopathies. The mechanisms behind these pathologies are poorly understood since the function of SelN remains elusive. However, previous results obtained in humans and more recently in zebrafish pointed to a potential role for SeIN during embryogenesis. Using qRT-PCR, Western blot and whole mount in situ hybridization, we characterized in detail the spatio-temporal expression pattern of the murine Sepn I gene during development, focusing particularly on skeletal muscles.

Results: In whole embryos, Sepn I transcripts were detected as early as E5.5, with expression levels peaking at EI2.5, and then strongly decreasing until birth. In isolated tissues, only mild transcriptional variations were observed during development, whereas a striking reduction of the protein expression was detected during the perinatal period. Furthermore, we demonstrated that Sepnl is expressed early in somites and restricted to the myotome, the sub-ectodermal mesenchyme and the dorsal root ganglia at mid-gestation stages. Interestingly, Sepn I deficiency did not alter somitogenesis in embryos, suggesting that SelN is dispensable for these processes in mouse.

Conclusion: We characterized for the first time the expression pattern of Sepnl during mammalian embryogenesis and we demonstrated that its differential expression is most likely dependent on major post-transcriptional regulations. Overall, our data strongly suggest a potential role for selenoprotein $\mathrm{N}$ from mid-gestation stages to the perinatal period. Interestingly, its specific expression pattern could be related to the current hypothesis that selenoprotein $\mathrm{N}$ may regulate the activity of the ryanodine receptors.
\end{abstract}




\section{Background}

Selenium is a rare trace element mainly present in biological systems as a selenocysteine ( $\mathrm{Sec}$ ), an amino acid found in proteins called selenoproteins and encountered in all lineages of life. Insertion of the Sec residue occurs through a recoded UGA stop codon which is recognized by a complex machinery involving a secondary structure present in the 3'UTR region of the mRNA (Sec Insertion Sequence, SECIS), which interacts during translation with specific factors, such as SBP2 (SECIS Binding Protein 2) and eEFSec (eukaryote Elongation Factor), leading to Sec insertion rather than termination $[1,2]$. In mammals, about 25 selenoproteins have been described [3]. Most of them are enzymes involved in oxidation-reduction reactions, with the selenocysteine residue(s) usually located in the catalytic site and conferring a strong enzymatic reactivity $[4,5]$. Moreover, most selenoproteins are expressed early during development $[2,6]$ and for several of them an essential role in embryogenesis has been established [79]. Selenium deficiency has been associated with different syndromes, such as Keshan cardiomyopathy, white muscle disease or rigid lamb syndrome, leading to dietary intake recommendations for humans and livestock [10]. Notably, mutations were identified in the SEPN1 gene, encoding selenoprotein N (SelN), as the genetic cause for Rigid Spine Muscular Dystrophy (RSMD1) [11]. This pathology is characterized by axial weakness, severe scoliosis usually requiring surgery, and respiratory insufficiency due to respiratory muscles weakness and necessitating mechanical nocturnal ventilation [12]. SEPN1 mutations were subsequently associated with three other neuromuscular disorders: the classical form of Multi-minicore Disease $(\mathrm{MmD})$ [13], rare cases of Desmin-Related Myopathy with Mallory Body-like Inclusions (MB-DRM) [14] and of Congenital Fibre Type Disproportion (CFTD) [15]. These four early onset autosomal recessive pathologies exhibit clinical and morphological overlaps; they are now grouped and termed SEPN1-related myopathies (SEPN1-RM).

We showed previously that SelN is a glycoprotein located in the membrane of the endoplasmic reticulum [16]. In humans, its expression appeared ubiquitous and was down-regulated during myoblast differentiation in culture, and in the transition from foetal to adult tissues [16]. In zebrafish, an early expression of sepn 1 was shown during embryogenesis, specifically in somites, the tail bud and the notochord $[6,17]$. Furthermore, sepn 1 zebrafish mutants obtained by morpholino injection, exhibited strong developmental defects such as tail malformations, disorganisation of somite borders, and abnormalities in muscles development and architecture [17,18]. These data, as well as the early onset of the human pathology, clearly pointed to a possible role for SelN during muscle development. Interestingly, physical and functional inter- actions between SelN and ryanodine receptors (RyR) were recently demonstrated, indicating that SelN may regulate RyR activity in muscles $[17,19]$.

Here, we characterized extensively the expression pattern of Sepn1 in mouse during pre- and post-natal development, in several tissues, particularly in skeletal muscles. We demonstrated that Sepn 1 is expressed early during mouse embryogenesis and that it is restricted to specific areas, including muscle precursors, at mid-gestation stages. In isolated tissues, mild variations of Sepn 1 transcripts were detected between ages, whereas the protein expression was strikingly down-regulated during the perinatal period, indicating post-transcriptional regulations of Sepn1 expression during development.

\section{Results \\ Spatio-temporal dynamics of Sepn I expression}

In whole embryos, Sepn1 expression was detected by qRTPCR as early as E5.5, with higher levels from E9.5 to E12.5 (six and ten fold increase, respectively). We then observed a five fold reduction of the expression from E12.5 to E18.5 (Figure 1A). This decrease was confirmed at the protein level by Western blot since SelN was reduced by more than two fold in E18.5 embryos compared to E12.5 (Figure $1 \mathrm{~B})$.

We then investigated Sepn1 expression in isolated quadriceps, diaphragm and non-skeletal-muscle tissues (brain, liver, kidney and heart) from late embryonic stages (E15, E18) to 15 months of age, with an earlier embryonic stage included for the heart (E13) (Figure 2A). By qRT-PCR, Sepn1 expression was detected in all tissues, albeit at barely detectable levels in liver (Figure 2A). Overall, variations in the mRNA levels observed for the different tissues were limited. Nevertheless, we observed a significant decrease in expression between E15 and E18 in the brain and in kidney. In parallel, increased levels were detected between E18 and postnatal day 1 in diaphragm, heart and kidney. Lastly, we showed a two fold increase in expression in the brain, between young (16 weeks) and adult mice (315 months) and a two fold reduction in kidney, between the first week of life and older mice (3 weeks to 15 months) (Figure 2A). Interestingly, expression in quadriceps and diaphragm did not exhibit the highest levels and appeared mostly constant. Similar results were obtained for skeletal muscles isolated from posterior (soleus + gastrocnemius) and anterior (tibialis anterior + extensor digitorum longus) hind limb compartments (data not shown).

We studied the expression of SelN at the protein level by Western blot, using proteins extracted from isolated tissues from E18 to 15 months of age. Several proteins were tested for protein loading control; actin and GAPDH 
A

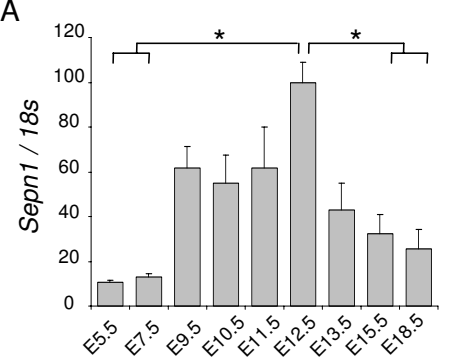

B

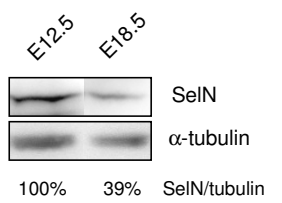

Figure I

Sepn I expression in whole embryos. A: Sepn I expression was quantified by QRT-PCR on CDNA from whole embryos between E5.5 and EI8.5. Normalization is performed on the 18s gene. Expression was detected as early as E5.5 and strongly increased until EI2.5. From this stage until birth, a striking decrease of the expression was observed. *, $\mathrm{p}<0.05$. B: Western blot analysis on $60 \mu \mathrm{g}$ of proteins from EI2.5 and El8.5 embryos was carried out using SelN and $\alpha$ tubulin (normalization) antibodies. SelN expression was reduced more than two fold at EI8.5 compared to EI2.5.

exhibited the least variability between the various developmental stages (Figure 2B and data not shown). Nevertheless, quadriceps homogenates displayed an increase of both actin and GAPDH expressions after 15 days of age; for this tissue, no ideal protein normalization could be obtained. For all the tissues considered except liver, we observed a striking reduction in SelN expression between E18 and postnatal day 1 (about three fold reduction), and even more so at adult ages when it became barely detectable (less than $10 \%$ compared to E18) (Figure 2B). In protein extracts from liver, SelN was never detected, even at E18 (Figure 2B), in keeping with the transcript quantifications.

In parallel, using mouse $\mathrm{C} 2 \mathrm{C} 12$ muscle cells, we observed a 50\% decrease of Sepn 1 expression in myotubes after 2 and 3 days of differentiation, compared to myoblasts (Figure $3 \mathrm{~A}$ ). This reduction was even more marked at the protein level with a five fold reduction detected in myotubes compared to myoblasts by Western blot (Figure 3B). This is in accordance with data previously obtained with human cells [16].

\section{Specificity of Sepn I probes}

Since no appropriate antibody was available for immunohistochemistry, we investigated Sepn1 expression pattern during early embryogenesis by whole mount in situ hybridization (ISH). The murine Sepn1 cDNA [GenBank: NM 029100], 3461 bp in size, contains 12 exons, the inframe UGA codon being located in exon 9, and leads to a protein of 557 residues (Figure 4A). In mouse, no sequence homology was found with the alternatively

spliced third exon described in human, since this exon corresponds to a primate specific Alu sequence [11]. Two Sepn1 probes ( $\operatorname{Pr} 1: 573$ bp and $\operatorname{Pr} 2: 696$ bp) were synthesized from PCR fragments corresponding to the 3 ' region of the cDNA (Figure 4A). The corresponding murine transcript regions shared more than $85 \%$ identity with the human sequence. We performed Northern blot analysis with Pr1 and Pr2 probes, using RNA extracted from cultured human fibroblasts from a control subject and a RSMD1 patient with SEPN1 mRNA degradation due to a premature termination codon (p.L482fs [20] Figure 4B). For both probes, one band corresponding to SEPN1 transcripts was observed in control RNA, but was undetectable in RNA extracted from the patient, thus validating the specificity of the probes (Figure 4B and data not shown). Using mouse RNA extracted from E12 and E18 whole embryos, we detected only one $3.4 \mathrm{~kb}$ band by Northern blot, which corresponds to the predicted transcript size for Sepn1 (Figure 4C).

\section{Expression pattern of Sepn I during embryogenesis}

Sepn1 deficient mouse embryos were used as negative controls for whole mount ISH. The recombinant gene construct introduced a frame-shift and a premature stop codon within the coding sequence, leading to a loss of the functional protein in mutants (Rederstorff et al., manuscript in preparation). As shown in Figure 5A, Sepn1 expression was barely detectable at the transcript levels in E12.5 mutant whole embryos (Sepn $1 \%$ ) compared to wild-type (Sepn $1^{+/+}$), indicative of the Sepn 1 mRNA degradation in the model. At the protein level, SelN was observed in wild-type embryos by Western blot but was undetectable in mutant samples (Figure 5B).

Identical expression patterns were obtained with $\operatorname{Pr} 1$ and Pr2; results presented in Figure 6 correspond to ISH performed with the Pr1 probe. At the different embryonic stages tested, no signal could be detected using either sense probes with wild-type embryos (Figure 6A, panel a and data not shown) or antisense probes with Sepn $1 \%$ embryos (Figure 6B, panel $\mathrm{i}$ and 6C, panel $\mathrm{t}$ ). This confirmed the specificity of the staining pattern obtained.

In E8.5 whole embryos, we detected Sepn 1 expression in somites and the neural tube, as well as in the mesenchyme located under the ectoderm, in the head and the branchial arches of embryos (Figure 6A, panels b-f). At E9.5, a ubiquitous-like staining was observed in whole embryos, with a stronger expression in somites (Figure 6B, panel g). Upon vibratome sectioning, the staining was associated with expression in the sub-ectodermal mesenchyme, as depicted in forebrain (Figure 6B, panel h). At E10.5 and E11.5, whole embryos appeared strongly stained in the dorsal part of the body, the limb buds, the branchial arches and the head (Figure 6C, panels j-m). Following 
A
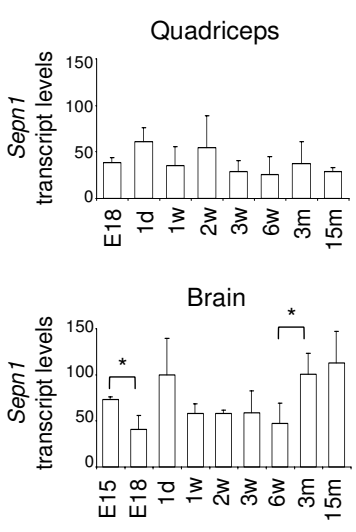

B
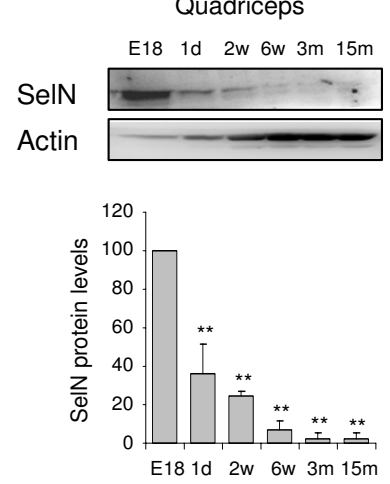

Brain
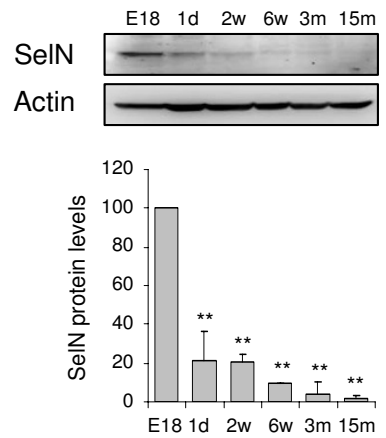
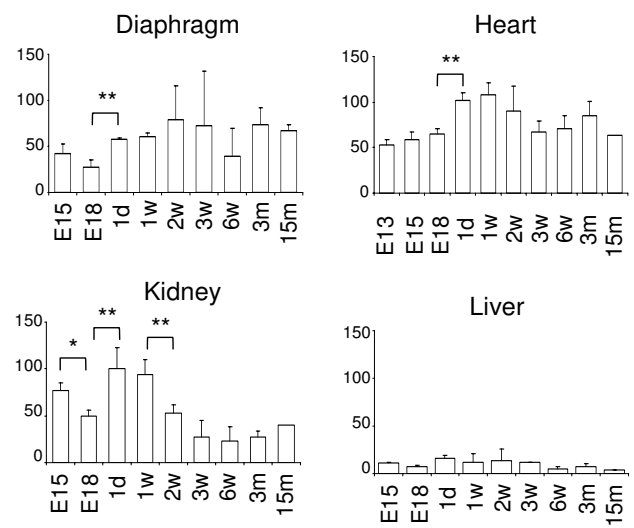

Diaphragm
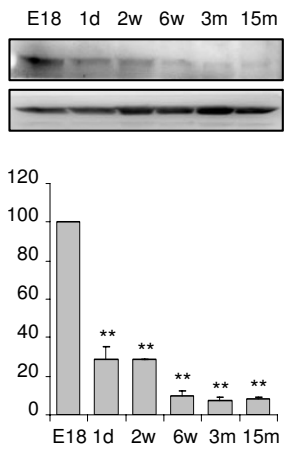

Kidney
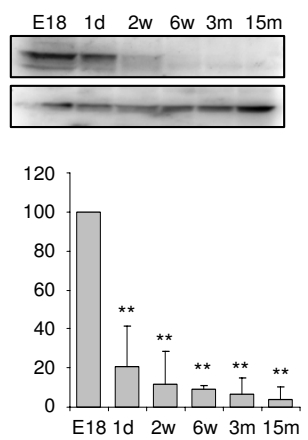
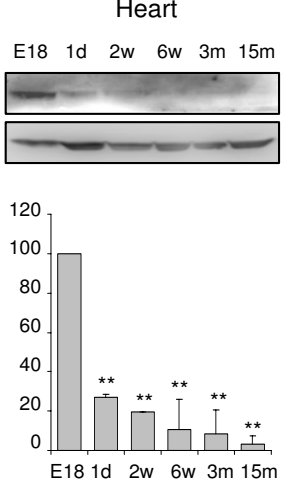

Liver

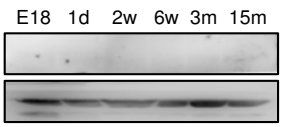

\section{Figure 2}

Expression of SepnI transcript and SeIN protein in isolated tissues between late embryonic and post-natal stages. A: Sepn I expression was quantified by qRT-PCR and normalized to the $18 \mathrm{~s}$ gene. Expression in quadriceps was quantified from EI 8 to 15 months; in diaphragm, liver, brain and kidney from EI5 to 15 months; in heart from EI 3 to 15 months. Only mild variations were detected, including between pre- and postnatal stages. Significant variations are indicated for two consecutive points: *, p < 0.05; **, p < 0.01. B: SelN Western blot analyses are shown for quadriceps, diaphragm, heart, liver, brain and kidney at stages EI8, postnatal day I, 2 and 6 weeks, 3 and 15 months. SelN was undetectable in liver. Actin was used as a reference. Quantifications were normalized on the expression measured at El8 and are presented below the blots. For all tissues, a striking reduction of the expression was detected between EI 8 and postnatal day I. This decrease was even more marked in the following post-natal stages. Statistical analyses were performed between EI 8 and all other stages (**, $p<0.01$ ). d: days, w: weeks, m: months. 
sectioning, we observed expression in the dorsal root ganglia (drg). Furthermore, within the somites, the myotome appeared strongly stained, whereas little to no signal was detected in the dermomyotome of embryos (Figure 6C, panels $\mathrm{n}-\mathrm{q}$ ). Moreover, as previously seen, the mesenchyme located under the ectoderm exhibited staining, particularly in the head and limb buds (Figure 6C, panels $r$, s), although this signal was weak and required a longer revelation time as compared to the staining observed in the myotome and drg.

Interestingly, at all of these stages, we did not observe staining in the heart by ISH, whereas Sepn 1 expression was detected as early as E13 in this tissue by qRT-PCR. This could result either from Sepn1 cardiac expression appearing after E11.5, or more likely from the higher sensitivity of the qPCR technique.

\section{Somitogenesis in Sepn I deficient murine embryos}

Since an early expression of Sepn1 was observed in somites and defects were reported in somite organisation and myogenic factors expression in sepn 1 morpholino injected zebrafish embryos $[17,18]$, we specifically investigated these events in Sepn $1 \%$ embryos. At mid-gestation stages (E10 and E11.5), ISH performed against the myogenic determination factors Myf5, MyoD, and Pax3 revealed no defect in somite size and organisation in Sepn $1 \%$ embryos compared to wild-type (Figure 7A and data not shown). Moreover, the expression pattern of these factors was similar in both types of embryos (Figure 7A). Likewise, the expression of myosin heavy chain, a marker of differentiated muscle cells, was unaltered when visualized by immunohistochemistry in E11.5 and E13 deficient embryos (Figure 7B). These data were confirmed by qRT-PCR and Western blot analyses that demonstrated no modification of the myogenic factors expression in the deficient embryos (Figure 7C and data not shown). Lastly, the expression patterns of Scleraxis, involved in the establishment of myotendinous junctions [21], and of TrkA, a neurotrophin receptor which was used as a marker of the drg [22], appeared unmodified in Sepn1\% embryos by whole-mount ISH (Figure 7A).

\section{Discussion}

In this study, we characterized for the first time the precise spatio-temporal expression pattern of the murine Sepn 1 gene, the homolog of SEPN1 involved in several forms of early onset myopathies in humans. We showed that Sepn1 is expressed early during embryogenesis and strongly down-regulated before birth. We also demonstrated that, in most isolated tissues, Sepn1 expression exhibited limited variations at the transcript levels between pre- and post-natal stages, but significant changes at the protein levels were detected, thereby pointing to a major posttranscriptional regulation of this gene. Of particular inter- est are some specific tissues where a significant increase in the expression of transcript was observed between E18 and postnatal day 1 (for kidney) or older mice (for the brain), whereas an almost $80 \%$ decrease in the protein levels was detected between equivalent stages. Despite the lack of data concerning the developmental expression pattern of Sec insertion factors, one regulatory mechanism, inherent to selenoproteins, may be based on the availability of these specific factors. Indeed, this complex machinery may exhibit spatial and temporal regulation, associated with a hierarchy in the synthesis of selenoproteins, leading to potential fine tuning of each selenoprotein in both physiological and selenium deprivation conditions [23-26]. It is thus conceivable that SelN expression is modulated depending on its requirements and biological roles in each tissue at the various developmental stages. Notably, we reported recently that the loss of interaction between the SECIS sequence and SBP2 abolished SelN translation and also led to the degradation of SEPN1 mRNA [20]. Therefore, modifying the interaction with the translational machinery may also affect Sepn 1 transcript stability in mouse. Hence, whether the regulation of SelN during development depends on the availability of Sec insertion factors remains questionable.

Another regulatory pathway could involve microRNAs, recently reported as major factors for translation modulation without mRNA expression perturbation [27]. Their importance in several developmental mechanisms has already been well established in most species, including vertebrates [28]. We determined that the Sepn 1 sequence is a potential target for several microRNAs (PC, PG, personal communication); however, their biological relevance and developmental involvement are not yet established. On the other hand, SelN expression may also be modulated by protein degradation or cleavage, as reported for different proteins located within the endoplasmic reticulum membrane [29]. Such proteolysis was suggested in the study of Deniziak et al. who observed a potential cleavage product by Western blot that correlates with reduced expression of SelN in zebrafish [18]. However, it was not investigated whether the degradation was physiological, or rather linked to the experimental conditions. Lastly, it is noteworthy that in some tissues, such as kidney and liver, in which similar dynamics were observed for protein and transcript expression, the gene is most likely regulated at the transcriptional level, although the factors involved are not known yet. Taken together, our findings reveal that complex transcriptional and translational regulation networks may be associated with the expression of SelN during development.

The expression pattern presented herein provides interesting insights into the physiological functions of SelN. Indeed, in all of the tissues examined except liver, high 
A

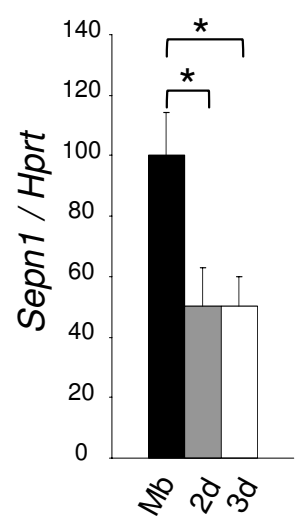

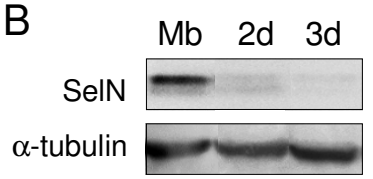

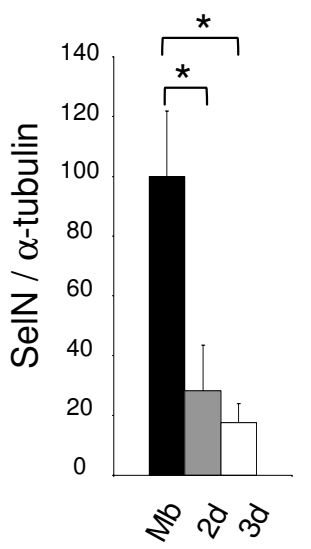

Figure 3

Sepn I expression during murine myoblast differentiation. A: Sepn I qRT-PCR on cDNA from $\mathrm{C} 2 \mathrm{Cl} 2$ myoblasts $(\mathrm{Mb})$ and myotubes after 2 and 3 days $(2 d / 3 d)$ in differentiation medium. Hprt served for normalization. A two fold reduction of Sepn I expression was observed in myotubes compared to undifferentiated cells. B: SelN Western blot analysis on proteins from $\mathrm{C} 2 \mathrm{Cl} 2$ cells (Mb, $2 \mathrm{~d}$, and $3 \mathrm{~d}$ ), normalized to $\alpha$-tubulin. Note that the decrease of SelN expression between myoblasts and myotubes is even more marked compared to transcript quantification. *, $\mathrm{p}<0.05$.

levels of SelN expression were limited to a particular temporal window, with a pronounced and progressive reduction observed between E18 and post-natal stages. These results are consistent with those obtained in zebrafish where a strong reduction of the expression was detected during somites differentiation $[17,18]$, and in humans where a decrease was observed between foetal and adult tissues [16]. This developmental pattern suggests that SelN may participate in the maturation of cells and organs from mid-gestation to perinatal stages. This notion is supported by the observation that SelN expression decreased during both human [16] and murine myoblast differentiation in culture. To our knowledge, there is no similar expression pattern reported for other selenoproteins but this may be at least partly due to the lack of studies focusing on the expression pattern of these proteins in mammals. Notwithstanding, in mouse, selenoprotein W (SelW) was found to be expressed early during development (E6) and to decrease during myoblast differentiation, but comparisons between pre- and post-natal stages in different tissues were not reported [7]. Moreover, in zebrafish, SelM was the only other selenoprotein reported to be expressed at the transcript levels in somites [6].

Based on the previous data obtained in humans and zebrafish, a potential link between SelN and cell proliferation has already been proposed [16]. At mid-gestation stages, we found that Sepn 1 is expressed in the mesenchyme located under the ectoderm. Taking into account our previous work showing high expression in fibroblasts [16], we attributed this expression to mesenchymal cells that share the general features of fibroblasts. Moreover, we first hypothesized that this pattern could correlate with a higher proportion of proliferative cells in this area. However, BrdU staining in E11.5 embryos did not reveal a higher mitotic activity in this region (data not shown). Likewise, in mature somites, Sepn1 expression was detected in the myotome, mainly composed of postmitotic cells that have initiated the myogenic program, while its expression was almost undetectable in the dermomyotome where most cells are proliferating [30]. Therefore, at least during mouse embryogenesis, there is no straightforward correlation between Sepn1 and cell proliferation.

The early expression detected in somites correlates with ISH results obtained in zebrafish [6]. Moreover, in mice, the restricted expression found in myotome indicates that Sepn1 is expressed by muscle precursors and maturating myocytes but less or not by uncommitted cells. Interestingly, in contrast to observations performed on zebrafish embryos knock-down for sepn1 expression [17,18], we did not detect any defects in somite organisation nor in myogenic factors expression, at mid-gestation stages, in Sepn1 mutant embryos. This suggests that SelN is dispensable for somitogenesis as well as the early waves of myogenesis in mouse. The simplest explanation would be that redundant activities exist in mouse, which may be less active in others animals. Due to the common reactivity of selenoproteins conferred by the Sec residue, one can hypothesize that some functional redundancy may exist within the protein family. Therefore, it would be interesting to further investigate other selenoproteins located in the endoplasmic reticulum, such as Sep15, SelS and SelM, and/or expressed predominantly during embryogenesis, like Gpx6 [2]. In addition, among the selenoproteins expressed in skeletal muscles, SelW is particularly interesting because of its dynamic expression during muscle maturation and its presumed association with white muscle disease [31]. Quantification of several selenoproteins expression by qRT-PCR revealed no difference between E12.5 wild-type and mutant Sepn $1^{-/}$whole embryos (data not shown). However, additional experiments, including older mice and protein analyses, need to be performed to resolve this issue.

Lastly, the barely detectable levels of SelN expression found in mature muscles fit with the previous observations in human tissues [16] and suggest a limited role for the protein at these stages. This contrasts with the progres- 
A

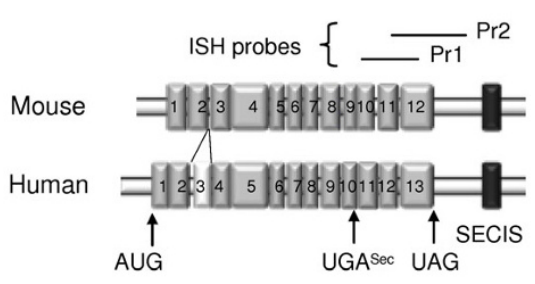

B

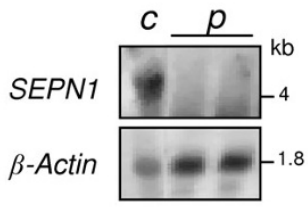

C

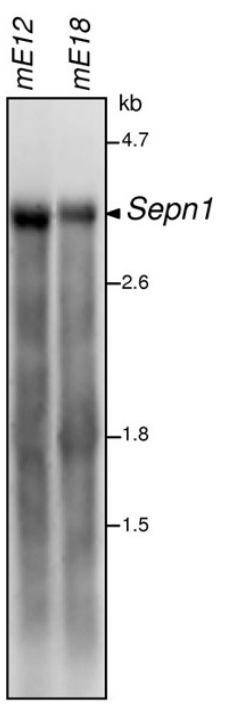

Figure 4

Specificity of the Sepn I probes. A: Schematic representation of the murine (Sepnl) and human (SEPNI) mRNA. Exons and the SECIS sequence are represented by grey and black boxes, respectively. The human third exon, corresponding to an Alu sequence, is represented by a white box. Target sequences of PrI and Pr2 ISH probes are shown above the schema. B: Northern blot analysis, using the PrI probe, of RNA extracted from human control fibroblasts (c) and fibroblasts from a RSMDI patient with a homozygous premature stop codon, leading to SEPNI mRNA degradation (p). -actin is used as a reference. A 4.2/4.3 kb band was observed in control fibroblasts but not in RSMD I fibroblasts, validating the specificity of PrI for SEPNI transcripts. C: Northern blot analysis of RNA from mouse EI2 (mE/2) and El8 ( $m E / 8$ ) whole embryos shows a single $3.4 \mathrm{~kb}$ band corresponding to SepnI transcript.

sive evolution of the pathology with age. One reason may be that SelN deficiency during muscle development leads to increased fragility of the tissue that worsens during aging. Another non necessarily exclusive hypothesis is that SelN is required in mature muscles subjected to constant tonic contractions, since the pathology affects more severely the respiratory and postural muscles. However, our data showing similar expression levels in muscles as different as quadriceps and diaphragm do not appear to sustain this hypothesis. The characterization of Sepn1 deficient mice, from later developmental stages to adult ages, will constitute a major point of investigation in order to better understand SelN function in muscle physiology and the remaining paradox regarding its almost ubiquitous expression and the specific muscular phenotypes.

Taking into account the recent studies suggesting a role for SelN in the regulation of ryanodine receptors (RyR) activity $[17,19]$, it is interesting to try and correlate their devel- opmental activity with the expression pattern of SelN described herein. RyR1 expression was reported in mouse myotome as early as E9.5 by ISH [32] but significant protein expression was only detected at late embryonic stages in rodents $[33,34]$. In addition, RyR1 expression greatly increases two weeks after birth and becomes predominant in mature muscles, therefore differing from SelN pattern [33-35]. In contrast, RyR3 expression strongly decreases at postnatal day 15 and it is almost undetectable in most adult tissues [33-36]. Furthermore, it was reported that its expression is exclusively maintained in highly active muscles, such as diaphragm or neck muscles [37] in which it may amplify calcium signals $[38,39]$. Interestingly, these tissues correspond to the most affected muscles in SEPN1related myopathies. Taken together, this comparison between RyR channels and SelN weakens the proposed functional link between RyR1 and SelN, but suggests a closer relationship between RyR3 and SelN. This is further substantiated by our ISH results. Indeed, Sepn1 staining was detected in the dorsal root ganglia which correspond to neural crest derivatives containing cell bodies of sensitive neurons, where a CICR mechanism was reported and associated with RyR3 channels $[40,41]$. We note also that Sepn1 was expressed in the sub-ectodermal mesenchyme, a region known to dynamically participate in cell migration during development, with active calcium waves involved in this process. Finally, the precise role of SelN in the regulation of RyR channels and the existence of other substrates remain largely unsolved.

\section{Conclusion}

This is the first study that establishes the precise spatiotemporal expression pattern of selenoprotein $\mathrm{N}$ in mammals, with both transcript and protein analyses at the different developmental stages. We showed that SelN is expressed early during mouse embryogenesis, particularly in somites and muscle precursors. Nevertheless, it appears to be dispensable for correct somitogenesis and expression of myogenic factors at mid-gestation stages. We also demonstrated that SelN expression pattern is wide-spread and is subject to post-transcriptional regulations which lead to the significant reduction of its protein expression levels during the perinatal period. Our results suggest important functions for SelN during the maturation of cells and organs. Further investigations should focus on the particular developmental window when SelN is strongly expressed, to determine the involvement of this selenoprotein in muscle physiology and to unveil the pathophysiological mechanisms leading to muscle alteration in SEPN1-related myopathies.

\section{Methods \\ Cell culture}

Mouse $\mathrm{C} 2 \mathrm{C} 12$ cells were grown in an humidified atmosphere of $5 \% \mathrm{CO}_{2}$, at $37^{\circ} \mathrm{C}$, in Dulbecco's modified Eagle's 


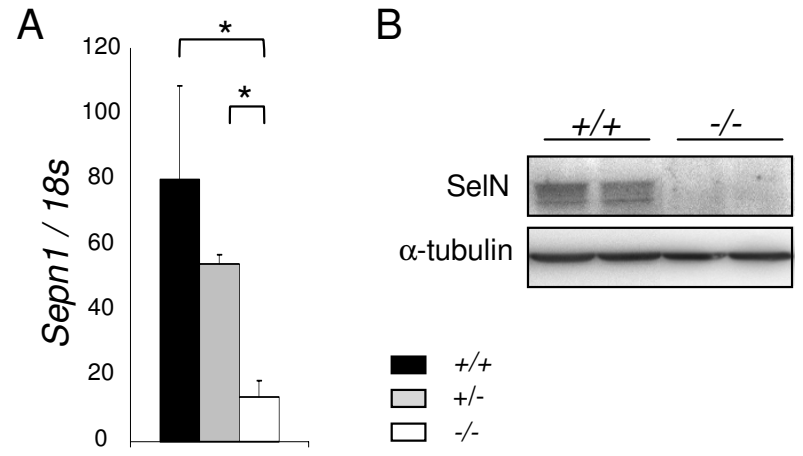

Figure 5

SelN deficiency in the murine model. Sepn I expression analyzed by qRT-PCR (A) and Western blot (B) performed on whole EI 2.5 littermate embryos: wild-type $(+/+)$, heterozygous (+/-) and homozygous mutants (-/-). I 8s gene and $\alpha-$ tubulin were used for normalization. Sepn I transcript expression was almost abolished in the homozygous mutants and SelN was undetectable by Western blot in these mice. *, $p<0.05$.

medium (DMEM, Invitrogen) supplemented with 20\% foetal calf serum (FCS, Invitrogen), $2 \mathrm{mM}$ gentamycine (Invitrogen). Differentiation into myotubes was induced by switching the medium to DMEM supplemented with $1 \%$ FCS.

\section{Mice and genotyping}

$\mathrm{C} 57 \mathrm{Bl} / 10$ mice were euthanized with isoflurane and tissues were dissected at various ages. Embryos were obtained from gestating females of the Sepn1 knock-out lineage (Rederstorff et al., article in preparation) between embryonic days 5.5 (E5.5) and 18.5 (E18.5), the morning of the vaginal plug indicating the stage E0.5. For in situ hybridization, embryos were dissected at $4{ }^{\circ} \mathrm{C}$ in phosphate buffer saline (PBS) and freed from their extraembryonic membranes. At E9.5 and older stages, the neural tube was pierced open at the level of the brain to facilitate exchange of solutions. Embryos were fixed overnight at $4{ }^{\circ} \mathrm{C}$, in $4 \%$ paraformaldehyde (PFA), dehydrated in methanol and conserved at $-20^{\circ} \mathrm{C}$. DNA extraction was carried out for genotyping with the Wizard Genomic DNA Isolation System (Promega) according to the manufacturer's recommendations. All animal studies were performed in accordance with the European Union guidelines for animal care and approved by the local ethical committee on animal experimentation.

\section{Total RNA extraction and first-strand CDNA synthesis}

Whole embryos and isolated tissues were frozen in liquid nitrogen. Total RNA was extracted using the RNeasy
Fibrous Tissue Mini Kit (Qiagen) according to the manufacturer's instructions. Total RNA was also extracted from cultured human fibroblasts (control and RSMD1 patient with a c.1446delC homozygous mutation) by Trizol (Invitrogen) procedure as previously described [11]. RNA yield and quality were assessed with the Nanodrop system (Thermo Scientific) and on the 2100 Bioanalyzer (Agilent Technologies). RNA samples with a RIN (RNA Integrity Number) superior to 8 were used for quantification. cDNA was synthesized from $500 \mathrm{ng}$ of RNA with random hexamer primers using the SuperScript First-Strand Synthesis System for RT-PCR (Invitrogen).

\section{Quantitative PCR (qPCR)}

Sepn1 transcripts were quantified by real time PCR using the following primers: 5'GCTTTCCTGTAGAGATGATG3'/ 5'GCCCCGCCGGAGTCCTTC3'. qPCR were performed on the LightCycler480 System (Roche) using the LighCycler480 SYBR Green I Master mix (Roche). The program included an initial denaturation step of $8 \mathrm{~min}$ at $95^{\circ} \mathrm{C}$, followed by 40 amplification cycles of denaturation at $95^{\circ} \mathrm{C}$ for $10 \mathrm{sec}$, hybridization at $63^{\circ} \mathrm{C}$ for $15 \mathrm{sec}$, and elongation at $72^{\circ} \mathrm{C}$ for 15 sec. Quantification of additional genes was performed similarly with the following primers: 18s: 5'ACCTGGTTGATCCTGCCAGT3'/5'CTCACCGGGTTGGTTTTGAT3'; Hprt:5'AAGCAGATGGCCACAGAACT3'/5'ACCCCACGAAGTGTTGG ATA 3'. Data were analyzed using the LightCycler480 analysis software (Roche).

\section{RNA probes synthesis and whole-mount In Situ Hybridization (ISH)}

Two digoxigenin (DIG) labeled riboprobes ( $\operatorname{Pr} 1 / \operatorname{Pr} 2)$ were generated from Sepn1 PCR fragments obtained from total murine cDNA with the following primers: Pr1: 5'TCCTAGATGAGGACGGCAAC3'/5'GGTCCTCAAAGCTGGATGA G3'; Pr2: 5'CGCCCATCCTCACTCTCCTC3'/5'TCCAGTCCACT CCCTAT TCACA3'. Fragments were cloned in the pGEM-T vector (Promega). Antisense and sense probes were synthesized using the DIG RNA Labeling Kit (Roche) with T7 or SP6 RNA polymerase. Sense probes were used as negative controls. Yield and quality of the probes were estimated by electrophoresis and dot blot compared to a standard $(\beta$ actin probe Roche). MyoD, Myf5, Scleraxis and TrkA probes, previously described $[42,43]$, were also used for whole mount ISH.

\section{Whole embryo In Situ Hybridization}

In situ hybridizations (ISH) were performed on embryos aged from E8.5 to E11.5, as previously described [43]. Briefly, after rehydration, embryos were treated with 10 $\mu \mathrm{g} / \mathrm{ml}$ proteinase $\mathrm{K}$ (Qiagen) at room temperature, from 10 to $30 \mathrm{~min}$ according to their age and fixed in 4\% PFA. Embryos were incubated for more than $1 \mathrm{~h}$ in hybridization buffer (50\% formamide, $1.3 \times \mathrm{SSC}$ pH 5, $50 \mu \mathrm{g} / \mathrm{ml}$ 
A

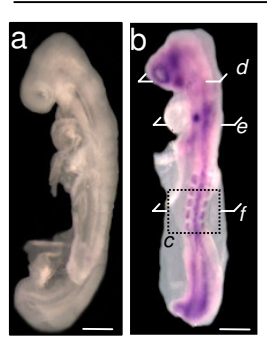

E8.5

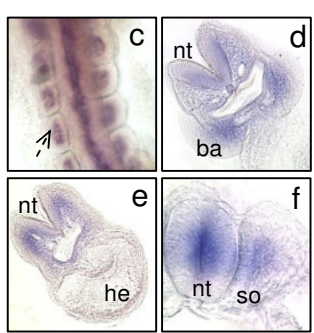

B

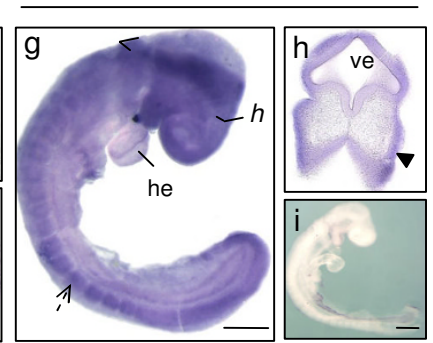

C

$\mathrm{E} 10.5 / \mathrm{E} 11.5$
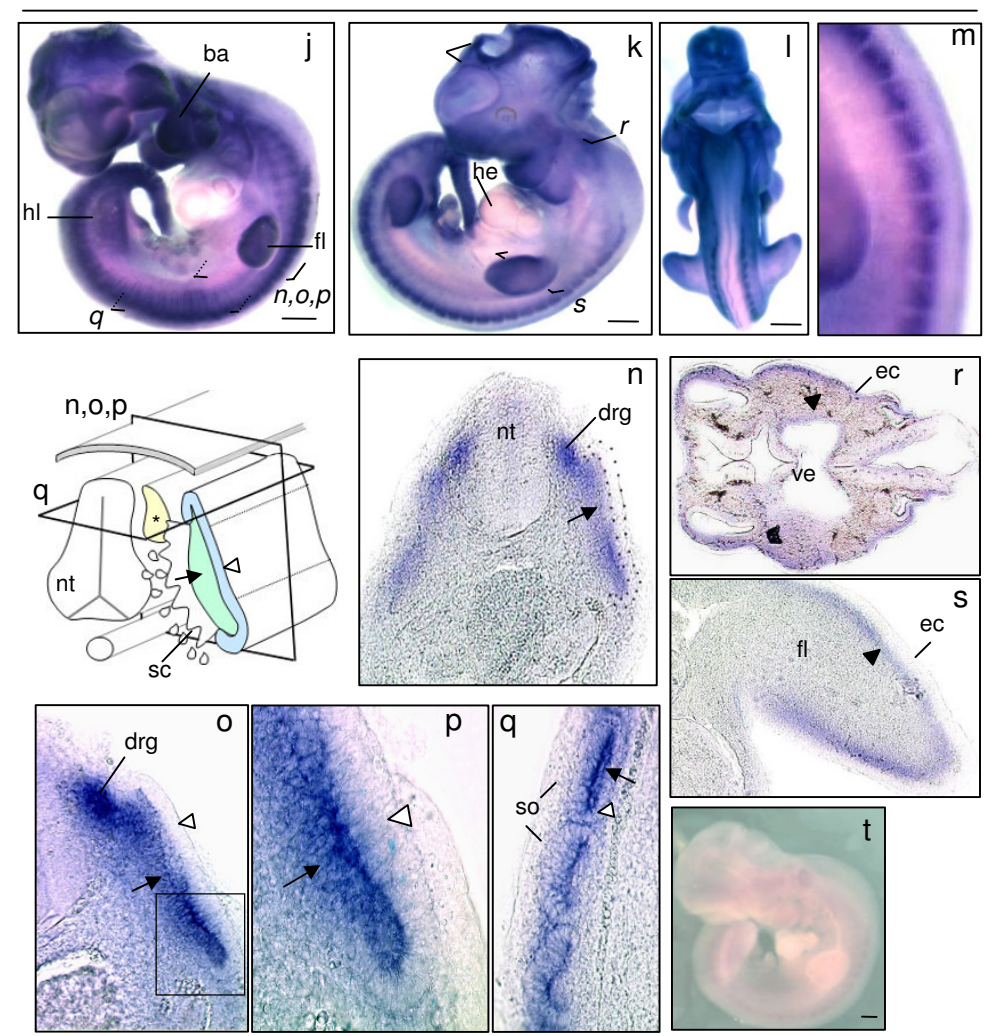

\section{Figure 6}

Sepn I whole mount in situ hybridization between E8.5 and EI I.5. A: E8.5 wild-type embryos hybridized with sense (a) and antisense (b-f) Sepn I PrI probes. No signal was detected with sense probes (a). With the antisense PrI probe, all the somites appeared strongly stained (broken arrow panel c). Sectioning with a vibratome revealed expression also in the branchial arches (d) and the neural tube (f). Note that no signal was observed in the heart (e). B: E9.5 wild-type (g, h) and Sepn I-l- (i) embryos hybridized with the SepnI antisense PrI probe. Broken arrow in (g) shows stained somites. Mesenchymal staining (black arrowhead) was revealed within vibratome sections (h: section in the forebrain). No signal was seen within the mutant embryos (i). C: EIO.5 (j) and EII.5 (k-m) wild-type embryos hybridized with the Sepn I antisense PrI probe. Dorsal region of wild-type embryos is shown in $(m)$. (n-s) High magnification of vibratome sections performed in the trunk $(n-q)$, the head ( $r$ ), and the limb bud (s). Inset p corresponds to a higher magnification of the outlined region in $o$. The section plans are indicated in the schema. Sepn I expression was detected in the sub-ectodermal mesenchyme (black arrowhead), the myotome (arrow) and the dorsal root ganglia (*). Note that overt staining was not observed in the dermomyotome (white arrowhead) nor in the heart. No signal was detected in Sepn I-l- embryos hybridized with Sepn I antisense probe (t). ba: branchial arches; drg: dorsal root ganglia (*); ec: ectoderm; fl: forelimb; he: heart; hl: hindlimb; nt: neural tube; so: somite; ve: ventricle. Scale bars in $(A)=200 \mu \mathrm{m}$, in $(B, C)=400 \mu \mathrm{m}$. 
yeast RNA, 5 mM EDTA pH 8, 0.2\% Tween-20, 0.5\% CHAPS, $100 \mu \mathrm{g} / \mathrm{ml}$ heparin) and then overnight with the appropriate probe at 0.5 to $2 \mu \mathrm{g} / \mathrm{ml}$, at $69^{\circ} \mathrm{C}$. Embryos were successively washed in hybridization buffer, in 1:1 hybridization buffer/MABT (100 mM maleic acid, 150 $\mathrm{mM} \mathrm{NaCl}, 1 \%$ Tween-20), and finally in MABT. After blocking in MABT, 2\% Blocking Reagent (Roche), 20\% inactivated goat serum (Invitrogen) for more than $1 \mathrm{~h}$ at room temperature, embryos were incubated overnight at $4{ }^{\circ} \mathrm{C}$ in alkaline phosphatase conjugated anti-DIG antibody (1:2000 Roche). Revelation was obtained with the BM Purple solution (Roche) at room temperature and/or at $4{ }^{\circ} \mathrm{C}$, during different time laps depending on the probe. Vibratome sections (50 to $80 \mu \mathrm{m}$ ) were performed after inclusion of the embryos in $10 \%$ sucrose overnight at $4{ }^{\circ} \mathrm{C}$ and then in $20 \%$ gelatin.

\section{Northern Blotting}

$30 \mu \mathrm{g}$ of total RNA were resolved on 1\% formaldehyde agarose gel and blotted overnight onto a positively charged nylon membrane (Roche). RNA were UV crosslinked to the membrane during $3 \mathrm{~min}$. Membranes were hybridized overnight at $68^{\circ} \mathrm{C}$ with diluted probe (100 ng/ $\mathrm{ml}$ ) in hybridization buffer (DIG Easy Hyb buffer Roche) and then rinsed in $2 \times$ SSC, $0.1 \%$ SDS at room temperature and in $0.1 \times \mathrm{SSC}, 0.1 \%$ SDS at $68^{\circ} \mathrm{C}$. Membranes were blocked in Blocking Reagent (Roche) diluted in $0.1 \mathrm{M}$ maleic acid, $0.15 \mathrm{M} \mathrm{NaCl}, \mathrm{pH} 7.5$, and then incubated with alkaline phosphatase conjugated anti-DIG antibody (Roche) in maleic acid buffer. After incubation in detection buffer (0.1 M Tris-HCl, 0.1 M NaCl, pH 9.5), signals were obtained with the CDP-star, ready-to-use buffer (Roche) on the ChemImager system (Alpha Innotech).

\section{Protein extraction and Western blot analysis}

Frozen tissues were ground in liquid nitrogen and homogenized in protein extraction buffer $(80 \mathrm{mM}$ Tris- $\mathrm{HCl}, \mathrm{pH}$ 6.8, 10\% SDS, $120 \mathrm{mM}$ sucrose, $10 \mathrm{mM}$ EDTA, $1 \mathrm{mM}$ PMSF and $1 \mathrm{mM}$ benzamidine). Homogenates were incubated $10 \mathrm{~min}$ at $55^{\circ} \mathrm{C}$, sonicated twice and quantified with the BCA Protein Assay (Pierce). $60 \mu \mathrm{g}$ of proteins were electrophoretically separated in $8 \%$ polyacrylamide SDS gel and transferred to a polyvinylidene difluoride membrane (Invitrogen). Membranes were immunoprobed with the following primary antibodies: rabbit anti-SelN (ab137 [16]), mouse anti-tubulin $\alpha$ (Sigma T5168), rabbit anti-actin (Sigma A2066), rabbit antiMyoD (Santa Cruz sc-304), rabbit anti-myogenin (Santa Cruz sc-576), rabbit anti-Myf5 (Santa Cruz sc-302) and mouse anti-MyHC (ATCC, MF20). They were then incubated with HRP-conjugated secondary antibodies (Dako). Signals were detected with chemiluminescent HRP substrate (Immobilon Western Millipore) on the SynGene instrument (Ozyme) and quantified with the ImageJ software.

\section{Immunohistochemistry}

After rehydration, E12.5 whole embryos were equilibrated in $15 \%$ sucrose overnight at $4^{\circ} \mathrm{C}$. They were then frozen in liquid nitrogen-cooled isopentane. Ten $\mu \mathrm{m}$ cryostat sections were fixed in $4 \% \mathrm{PFA}$, rinsed in $0.1 \mathrm{M}$ glycine, and incubated in methanol at $-20^{\circ} \mathrm{C}$. They were then placed in boiling $0.01 \mathrm{M}$ citric acid for $10 \mathrm{~min}$ to achieve antigen retrieval. Sections were blocked in 3\% IgG free bovine serum albumin (BSA Jackson ImmunoResearch) overnight at $4^{\circ} \mathrm{C}$ and with ChromoPure Mouse IgG, Fab Fragments (Jackson ImmunoResearch) for $30 \mathrm{~min}$. Sections were then incubated in 3\% IgG free BSA, $0,1 \%$ Triton X100 , with primary antibodies (MyHC: monoclonal mouse ATCC MF20-1/100; laminin: polyclonal rabbit Abcam ab11575-1/1000) for $3 \mathrm{~h}$ at room temperature. Alexa Fluor secondary antibodies (goat anti-mouse A568, goat anti-rabbit A488; Jackson ImmunoResearch) were incubated on slides for $90 \mathrm{~min}$ at room temperature. Sections were rinsed, mounted in Vectashield DAPI (Vector) and

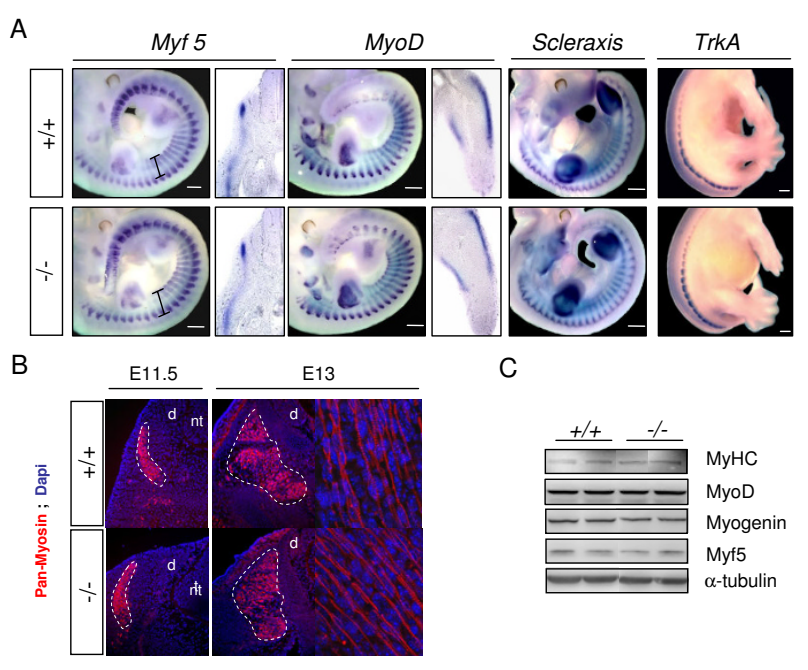

Figure 7

Somitogenesis in wild-type and Sepn $\mathrm{I}^{-/-}$embryos. A: Whole mount in situ hybridization, and corresponding vibratome sections, against Myf5, MyoD, Scleraxis (EI I.5) and TrkA (EI3) performed on Sepn I+/+ and Sepn I-/- embryos. No difference was observed in somite organisation and size (black fragments) between wild-type and mutants embryos. The expression pattern of the tested factors appeared unaltered in absence of SelN. Vibratome sections correspond to the trunk level for Myf5 and to the limb bud for MyoD. B: Immunostaining against myosin heavy chain on EI I.5 and EI 3 wild-type and deficient embryos. The expression patterns were identical between the embryos. Myotome is outlined with a white broken line. C: Western blot analysis against myogenic factors, performed on proteins from wild-type and deficient EI 2.5 whole embryos, revealed no alteration of their expression in the mutant embryos. $\alpha$-tubulin was used for normalization. Scale bars $=400 \mu \mathrm{m}$. d: drg; nt: neural tube. 
observed with an Axiophot microscope (Zeiss). Images were captured using the Metaview software (Ropper Scientific).

\section{Statistical analyses}

Results represent Means \pm SD. Comparisons between two groups were performed using a Student's $t$-test or a MannWhitney rank sum test depending on the normal distribution of data, with a 0.05 level of confidence accepted for statistical significance. Multiple group comparison was performed by one way ANOVA with Dunn's pairwise procedures $(\mathrm{p}<0.05)$.

\section{Abbreviations}

CFTD: Congenital Fibre Type Disproportion; CICR: Calcium-Induced Calcium Released; drg: dorsal root ganglia; eEFSec: eukaryote Elongation Factor; ISH: In situ Hybridization; MB-DRM: Desmin-Related Myopathy with Mallory Body-like inclusions; $M m D$ : Multi-minicore Disease; qRT-PCR: quantitative Reverse Transcription Polymerase Chain Reaction; RSMD1: Rigid Spine Muscular Dystrophy; RyR: Ryanodine Receptor; SBP2: SECIS Binding Protein 2; SD: Standard Deviation; Sec: Selenocystein; SECIS: Sec Insertion Sequence; SelN: Selenoprotein N; SEPN1RM: SEPN1 Related-Myopathies.

\section{Authors' contributions}

PC carried out the qPCR, western blotting and in situ hybridization, analyzed and interpreted the data and drafted the manuscript. SM performed ISH probes synthesis. CG participated to the qPCR studies, and purified SelN antibodies. MR, AL and AK constructed and provided the deficient murine model. ST helped on ISH procedures and analysis of the results. VA and PG conceived and coordinated the overall study and helped draft the manuscript. All authors read and approved the final manuscript.

\section{Acknowledgements}

We would like to thank Dr. D. Duprez and Dr. P. Maire for the scleraxis and trkA probes, Dr. A. Jory for ISH technical assistance, Dr. N. Clarke and Dr. AC. Durieux for genotyping and fruitful discussions, and the Plate-forme Post-génomique de la Pitié-Salpêtrière. This work was supported by the Institut National de la Santé et de la Recherche Médicale (INSERM), the Association Française contre les Myopathies (AFM) and by the University Pierre et Marie Curie (UPMC). ST was funded by the Institut Pasteur. PC received $\mathrm{PhD}$ fellowships from the Ministère de la recherche et de l'enseignement, UPMC and AFM (I34I9).

\section{References}

I. Allmang C, Krol A: Selenoprotein synthesis: UGA does not end the story. Biochimie 2006, 88: I56I-7I.

2. Papp LV, Lu J, Holmgren A, Khanna KK: From selenium to selenoproteins: synthesis, identity, and their role in human health. Antioxid Redox Signal 2007, 9:775-806.

3. Kryukov GV, Castellano S, Novoselov SV, Lobanov AV, Zehtab O, Guigo R, Gladyshev VN: Characterization of mammalian selenoproteomes. Science 2003, 300:1439-43.
4. Jacob C, Giles GI, Giles NM, Sies H: Sulfur and selenium: the role of oxidation state in protein structure and function. Angew Chem Int Ed Engl 2003, 42:4742-58.

5. Lu J, Holmgren A: Selenoproteins. J Biol Chem 2009, 284:723-7.

6. Thisse C, Degrave A, Kryukov GV, Gladyshev VN, Obrecht-Pflumio S, Krol A, Thisse B, Lescure A: Spatial and temporal expression patterns of selenoprotein genes during embryogenesis in zebrafish. Gene Expr Patterns 2003, 3:525-32.

7. Loflin J, Lopez N, Whanger PD, Kioussi C: Selenoprotein W during development and oxidative stress. J Inorg Biochem 2006, 100:1679-84.

8. Bosl MR, Takaku K, Oshima M, Nishimura S, Taketo MM: Early embryonic lethality caused by targeted disruption of the mouse selenocysteine tRNA gene (Trsp). Proc Natl Acad Sci USA 1997, 94:553|-4.

9. Jakupoglu C, Przemeck GK, Schneider M, Moreno SG, Mayr N, Hatzopoulos AK, de Angelis MH, Wurst W, Bornkamm GW, Brielmeier $M$, et al.: Cytoplasmic thioredoxin reductase is essential for embryogenesis but dispensable for cardiac development. Mol Cell Biol 2005, 25:1980-8.

10. Rederstorff $M$, Krol A, Lescure A: Understanding the importance of selenium and selenoproteins in muscle function. Cell Mol Life Sci 2006, 63:52-9.

II. Moghadaszadeh B, Petit N, Jaillard C, Brockington M, Roy SQ, Merlini L, Romero N, Estournet B, Desguerre I, Chaigne D, et al.: Mutations in SEPNI cause congenital muscular dystrophy with spinal rigidity and restrictive respiratory syndrome. Nat Genet 200I, 29:17-8.

12. Moghadaszadeh B, Topaloglu H, Merlini L, Muntoni F, Estournet B, Sewry C, Naom I, Barois A, Fardeau M, Tome FM, et al.: Genetic heterogeneity of congenital muscular dystrophy with rigid spine syndrome. Neuromuscul Disord 1999, 9:376-82.

13. Ferreiro A, Quijano-Roy S, Pichereau C, Moghadaszadeh B, Goemans $\mathrm{N}$, Bonnemann $\mathrm{C}$, Jungbluth $\mathrm{H}$, Straub V, Villanova M, Leroy JP, et al.: Mutations of the selenoprotein $\mathbf{N}$ gene, which is implicated in rigid spine muscular dystrophy, cause the classical phenotype of multiminicore disease: reassessing the nosology of early-onset myopathies. Am J Hum Genet 2002, 7 I:739-49.

14. Ferreiro A, Ceuterick-de Groote C, Marks JJ, Goemans N, Schreiber G, Hanefeld F, Fardeau M, Martin JJ, Goebel HH, Richard P, et al.: Desmin-related myopathy with Mallory body-like inclusions is caused by mutations of the selenoprotein $\mathbf{N}$ gene. Ann Neurol 2004, 55:676-86.

15. Clarke NF, Kidson W, Quijano-Roy S, Estournet B, Ferreiro A, Guicheney P, Manson JI, Kornberg AJ, Shield LK, North KN: SEPN I: associated with congenital fiber-type disproportion and insulin resistance. Ann Neurol 2006, 59:546-52.

16. Petit N, Lescure A, Rederstorff M, Krol A, Moghadaszadeh B, Wewer UM, Guicheney P: Selenoprotein N: an endoplasmic reticulum glycoprotein with an early developmental expression pattern. Hum Mol Genet 2003, I 2: 1045-53.

17. Jurynec MJ, Xia R, Mackrill JJ, Gunther D, Crawford T, Flanigan KM, Abramson J], Howard MT, Grunwald DJ: Selenoprotein $\mathbf{N}$ is required for ryanodine receptor calcium release channel activity in human and zebrafish muscle. Proc Natl Acad Sci USA 2008, 105: 12485-90.

18. Deniziak M, Thisse C, Rederstorff M, Hindelang C, Thisse B, Lescure $A$ : Loss of selenoprotein $\mathbf{N}$ function causes disruption of muscle architecture in the zebrafish embryo. Exp Cell Res 2007, 313:156-67.

19. Arbogast S, Beuvin M, Fraysse B, Zhou H, Muntoni F, Ferreiro A: Oxidative stress in SEPN I-related myopathy: From pathophysiology to treatment. Ann Neurol 2009, 65:677-686.

20. Allamand V, Richard P, Lescure A, Ledeuil C, Desjardin D, Petit N, Gartioux C, Ferreiro A, Krol A, Pellegrini N, et al.: A single homozygous point mutation in a 3 'untranslated region motif of selenoprotein N mRNA causes SEPN I-related myopathy. EMBO Rep 2006, 7:450-4.

21. Murchison ND, Price BA, Conner DA, Keene DR, Olson EN, Tabin C], Schweitzer R: Regulation of tendon differentiation by scleraxis distinguishes force-transmitting tendons from muscleanchoring tendons. Development 2007, 134:2697-708.

22. White FA, Silos-Santiago I, Molliver DC, Nishimura M, Phillips H, Barbacid M, Snider WD: Synchronous onset of NGF and TrkA survival dependence in developing dorsal root ganglia. J Neurosci 1996, 16:4662-72. 
23. Driscoll DM, Copeland PR: Mechanism and regulation of selenoprotein synthesis. Annu Rev Nutr 2003, 23:17-40.

24. Kelly VP, Suzuki T, Nakajima O, Arai T, Tamai Y, Takahashi S, Nishimura S, Yamamoto M: The distal sequence element of the selenocysteine tRNA gene is a tissue-dependent enhancer essential for mouse embryogenesis. Mol Cell Biol 2005, 25:3658-69.

25. Berry MJ: Insights into the hierarchy of selenium incorporation. Nat Genet 2005, 37: I 162-3.

26. Carlson BA, Xu XM, Gladyshev VN, Hatfield DL: Selective rescue of selenoprotein expression in mice lacking a highly specialized methyl group in selenocysteine tRNA. J Biol Chem 2005 280:5542-8

27. Filipowicz W, Bhattacharyya SN, Sonenberg N: Mechanisms of post-transcriptional regulation by microRNAs: are the answers in sight? Nat Rev Genet 2008, 9:102-14.

28. Stefani G, Slack FJ: Small non-coding RNAs in animal development. Nat Rev Mol Cell Biol 2008, 9:2 19-30.

29. Brown MS, Ye J, Rawson RB, Goldstein JL: Regulated intramembrane proteolysis: a control mechanism conserved from bacteria to humans. Cell 2000, 100:391-8.

30. Tajbakhsh S, Buckingham M: The birth of muscle progenitor cells in the mouse: spatiotemporal considerations. Curr Top Dev Biol 2000, 48:225-68.

31. Lescure A, Rederstorff M, Krol A, Guicheney P, Allamand V: Selenoprotein function and muscle disease. Biochim Biophys Acta 2009 in press.

32. Rosemblit N, Moschella MC, Ondriasa E, Gutstein DE, Ondrias K, Marks AR: Intracellular calcium release channel expression during embryogenesis. Dev Biol I999, 206:163-77.

33. Bertocchini F, Ovitt CE, Conti A, Barone V, Scholer HR, Bottinelli R, Reggiani C, Sorrentino V: Requirement for the ryanodine receptor type 3 for efficient contraction in neonatal skeletal muscles. EMBO J I997, 16:6956-63.

34. Flucher BE, Conti A, Takeshima H, Sorrentino V: Type 3 and type I ryanodine receptors are localized in triads of the same mammalian skeletal muscle fibers. I Cell Biol I999, 146:621-30.

35. Kyselovic J, Leddy JJ, Ray A, Wigle J, Tuana BS: Temporal differences in the induction of dihydropyridine receptor subunits and ryanodine receptors during skeletal muscle development. J Biol Chem 1994, 269:2 I770-7.

36. Tarroni P, Rossi D, Conti A, Sorrentino V: Expression of the ryanodine receptor type 3 calcium release channel during development and differentiation of mammalian skeletal muscle cells. J Biol Chem 1997, 272:19808-13.

37. Rossi D, Murayama T, Manini I, Franci D, Ogawa Y, Sorrentino V: Expression and functional activity of ryanodine receptors (RyRs) during skeletal muscle development. Cell Calcium 2007. 4I:573-80.

38. Weisleder N, Ferrante C, Hirata Y, Collet C, Chu Y, Cheng H, Takeshima H, Ma J: Systemic ablation of RyR3 alters Ca2+ spark signaling in adult skeletal muscle. Cell Calcium 2007, 42:548-55.

39. Ikemoto T, Komazaki S, Takeshima H, Nishi M, Noda T, lino M, Endo M: Functional and morphological features of skeletal muscle from mutant mice lacking both type $I$ and type 3 ryanodine receptors. I Physiol 1997, 50 I (Pt 2):305- 12.

40. Ouyang K, Zheng H, Qin X, Zhang C, Yang D, Wang X, Wu C, Zhou $\mathrm{Z}$, Cheng $\mathrm{H}: \mathrm{Ca2}+$ sparks and secretion in dorsal root ganglion neurons. Proc Natl Acad Sci USA 2005, 102: I 2259-64.

4I. Lokuta AJ, Komai H, McDowell TS, Valdivia HH: Functional properties of ryanodine receptors from rat dorsal root ganglia. FEBS Lett 2002, 5 I I:90-6.

42. Tajbakhsh S, Rocancourt D, Cossu G, Buckingham M: Redefining the genetic hierarchies controlling skeletal myogenesis: Pax3 and Myf-5 act upstream of MyoD. Cell 1997, 89:127-38.

43. Edom-Vovard F, Bonnin M, Duprez D: Fgf8 transcripts are located in tendons during embryonic chick limb development. Mech Dev 200I, 108:203-6.

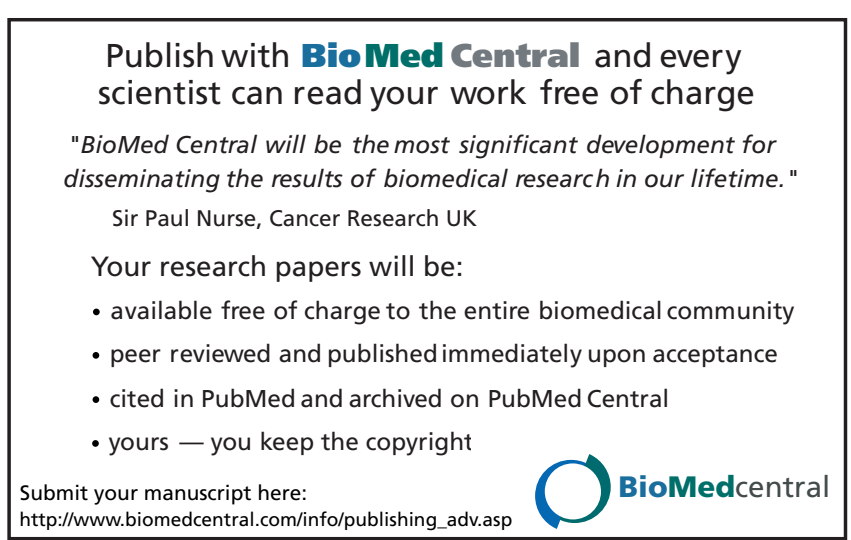

